\title{
Crystal structure of diethylenetriaminesilver(I) perchlorate, $\mathrm{Ag}\left(\mathrm{C}_{4} \mathrm{H}_{13} \mathrm{~N}_{3}\right)\left(\mathrm{ClO}_{4}\right)$
}

\author{
H.-L. Zhu* ${ }^{*, I I I}$, S.-C. Shao ${ }^{\mathrm{I}}$, J.-L. Ma ${ }^{\mathrm{I}}$, X.-Y. Qiu${ }^{\mathrm{I}}$, S. Yang ${ }^{\mathrm{I}}$ and L. Sun ${ }^{\mathrm{I}}$ \\ I Fuyang Normal College, Department of Chemistry, Fuyang, Anhui, 236041 P. R. China \\ II Wuhan University of Science and Engineering, Department of Chemistry, Wuhan, 430073 P. R. China
}

Received July 30, 2003, accepted and available on-line October 31, 2003; CCDC-No. 1267/1128

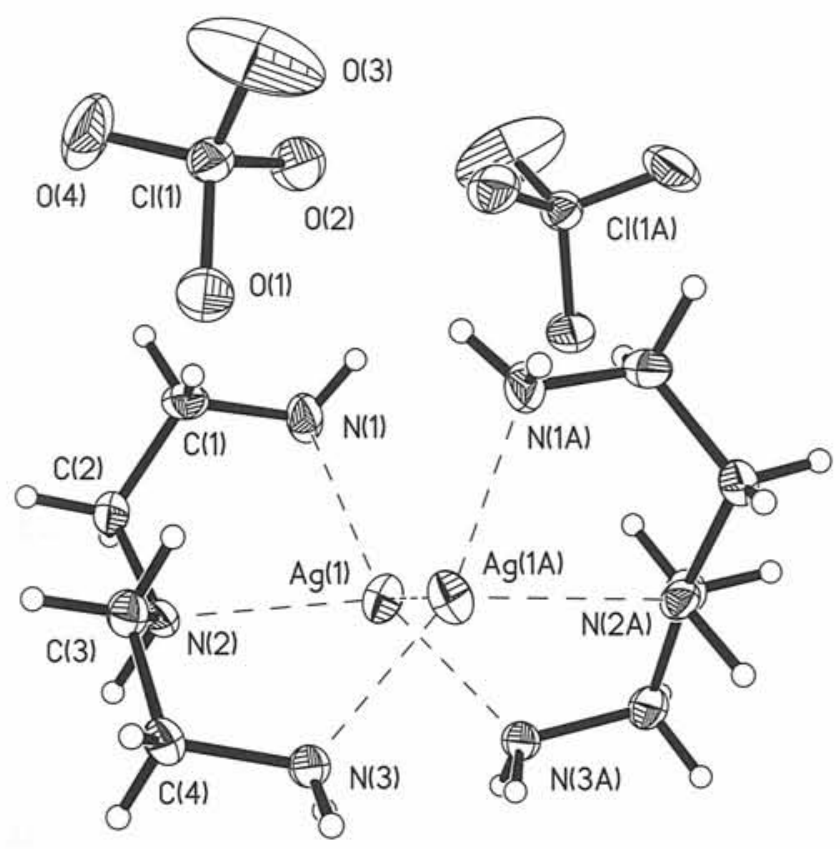

Abstract

$\mathrm{C}_{4} \mathrm{H}_{13} \mathrm{AgClN}_{3} \mathrm{O}_{4}$, orthorhombic, Pccn (No. 56), $a=17.730$ (7) $\AA$, $b=7.162(3) \AA, c=15.891(7) \AA, V=2017.9 \AA^{3}, Z=8$, $R_{\mathrm{gt}}(F)=0.047, w R_{\text {ref }}\left(F^{2}\right)=0.152, T=298 \mathrm{~K}$.

\section{Source of material}

With stirring to a dien ( $1 \mathrm{mmol}, 0.10 \mathrm{~g}$ ) solution in $30 \%$ ammonium solution $(5 \mathrm{ml})$ was added $\mathrm{AgClO}_{4}(1 \mathrm{mmol}, 0.20 \mathrm{~g})$ solution in $30 \%$ ammonium solution ( $5 \mathrm{ml}$ ), where dien is diethylenetriamine. The resulting clear solution was stood still in air for overnight. Colorless prism crystals suitable for X-ray determination were deposited at the bottom of the flask. The product was washed with water/methanol $(1: 1, \mathrm{v} / \mathrm{v})$ twice, and dried in vacuum under $\mathrm{CaCl}_{2}$ (yield 95\%). Elemental analysis: found - $\mathrm{C}$, $15.5 \% ; \mathrm{H}, 4.3 \% ; \mathrm{N}, 13.0 \% ; \mathrm{C}_{4} \mathrm{H}_{13} \mathrm{~N}_{3} \mathrm{O}_{4} \mathrm{ClAg}$ requires $\mathrm{C}, 15.5 \%$; $\mathrm{H}, 4.2 \% ; \mathrm{N}, 13.5 \%$.

\section{Experimental details}

The $\mathrm{H}$ atom attached to the secondary nitrogen atom was located from difference Fourier maps and refined isotropically. All the other hydrogen atoms were added on calculated positions.

\section{Discussion}

The title complex crystallizes primarily in a mononuclear form, in which the silver atom is coordinated in a Y-shape fashion

$\left[\angle \mathrm{N} 1-\mathrm{Ag} 1-\mathrm{N} 2=76.0(2)^{\circ}, \angle \mathrm{N} 2-\mathrm{Ag} 1-\mathrm{N} 3 \mathrm{~A}=126.9(2)^{\circ}\right.$ and $\left.\angle \mathrm{N} 1-\mathrm{Ag} 1-\mathrm{N} 3 \mathrm{~A}=157.0(3)^{\circ}\right]$ with the coordination of two nitrogen atoms from a dien and the third coordinate $\mathrm{N}$ atom from another dien. In each dien molecule, three nitrogen atoms respectively coordinate to two different $\mathrm{Ag}$ atoms with two $\mathrm{N}$ atoms joining one $\mathrm{Ag}$ atom and one end-nitrogen atom joining a different silver atom, forming a dimer. The $\mathrm{Ag} \cdots \mathrm{Ag}$ distance $3.035(2) \AA$ in the dimer is shorter than the van der Waals radii of two silver atoms ( $3.44 \AA)$, indicating some weak metal-metal interaction. The $\mathrm{Ag}-\mathrm{N}$ distances of silver and two primary amine nitrogens $(d(\mathrm{Ag} 1-\mathrm{N} 1)=2.200(7) \AA$ and $d(\mathrm{Ag} 1-\mathrm{N} 3 \mathrm{~A})=$ 2.166(6) $\AA$ ) are comparable to those found in other Ag-amine containing complexes. However, the $\mathrm{Ag}-\mathrm{N}$ (secondary amine) distance $(d(\mathrm{Ag} 1-\mathrm{N} 2)=2.543(6) \AA)$ is much longer than those in the similar complexes we reported previously. Weak hydrogen contacts (from 2.962 (4) $\AA$ to 3.348 (8) $\AA$ ) between nitrogen atoms from diens and oxygen atoms from perchlorates link the discrete dimers along $b$-axis into a one-dimensional chain.

Table 1. Data collection and handling.

Crystal:

Wavelength:

$\mu$ :

Diffractometer, scan mode:

$2 \theta_{\max }:$

$N(h k l)_{\text {measured, }} N(h k l)_{\text {unique: }}$ Criterion for $I_{\mathrm{obs}}, N(h k l) \mathrm{gt}$ :

$N(\text { param })_{\text {refined: }}$

Programs:

Table 2. Atomic coordinates and displacement parameters (in $\AA^{2}$ ).

\begin{tabular}{lllcll}
\hline Atom & Site & $x$ & $y$ & $z$ & $U_{\text {iso }}$ \\
\hline $\mathrm{H}(1 \mathrm{~A})$ & $8 e$ & 0.7275 & 0.0357 & 0.198 & 0.108 \\
$\mathrm{H}(1 \mathrm{~B})$ & $8 e$ & 0.6953 & -0.1398 & 0.2271 & 0.108 \\
$\mathrm{H}(3 \mathrm{C})$ & $8 e$ & 0.6895 & 0.3471 & 0.4955 & 0.072 \\
$\mathrm{H}(3 \mathrm{D})$ & $8 e$ & 0.6872 & 0.5489 & 0.4977 & 0.072 \\
$\mathrm{H}(1 \mathrm{C})$ & $8 e$ & 0.5977 & -0.0024 & 0.1763 & 0.087 \\
$\mathrm{H}(1 \mathrm{D})$ & $8 e$ & 0.6246 & 0.1911 & 0.2114 & 0.087 \\
$\mathrm{H}(2 \mathrm{~A})$ & $8 e$ & 0.5227 & 0.0721 & 0.2855 & 0.092 \\
$\mathrm{H}(2 \mathrm{~B})$ & $8 e$ & 0.5723 & -0.099 & 0.3126 & 0.092 \\
$\mathrm{H}(3 \mathrm{~A})$ & $8 e$ & 0.5278 & 0.345 & 0.3548 & 0.075 \\
$\mathrm{H}(3 \mathrm{~B})$ & $8 e$ & 0.6081 & 0.3908 & 0.3194 & 0.075 \\
$\mathrm{H}(4 \mathrm{~A})$ & $8 e$ & 0.5811 & 0.5714 & 0.4387 & 0.082 \\
$\mathrm{H}(4 \mathrm{~B})$ & $8 e$ & 0.5725 & 0.3892 & 0.4925 & 0.082 \\
$\mathrm{H}(1)$ & $8 e$ & $0.571(3)$ & $0.080(8)$ & $0.420(4)$ & $0.03(2)$ \\
& & & & &
\end{tabular}

colorless prism, size $0.13 \times 0.17 \times 0.28 \mathrm{~mm}$ Mo $K_{\alpha}$ radiation $(0.71073 \AA)$ $22.52 \mathrm{~cm}^{-1}$

Bruker SMART CCD, $\varphi / \omega$

$53.08^{\circ}$

10599,2030

$I_{\mathrm{obs}}>2 \sigma\left(I_{\mathrm{obs}}\right), 815$

122

SHELXTL [2], SHEXTL-plus [3]

\footnotetext{
* Correspondence author (e-mail: hlzhu@ wist.edu.cn)
} 
Table 3. Atomic coordinates and displacement parameters (in $\AA^{2}$ ).

\begin{tabular}{|c|c|c|c|c|c|c|c|c|c|c|}
\hline Atom & Site & $x$ & $y$ & $z$ & $U_{11}$ & $U_{22}$ & $U_{33}$ & $U_{12}$ & $U_{13}$ & $U_{23}$ \\
\hline $\operatorname{Ag}(1)$ & $8 e$ & $0.73904(4)$ & $0.0399(1)$ & $0.36349(5)$ & $0.0615(5)$ & $0.0741(6)$ & $0.0844(6)$ & $-0.0079(4)$ & $-0.0149(4)$ & $-0.0022(4)$ \\
\hline $\mathrm{Cl}(1)$ & $8 e$ & $0.6341(1)$ & $0.5440(4)$ & $0.0932(2)$ & $0.064(2)$ & $0.073(2)$ & $0.057(2)$ & $0.010(1)$ & $-0.000(1)$ & $0.003(1)$ \\
\hline $\mathrm{N}(1)$ & $8 e$ & $0.6961(4)$ & $-0.016(1)$ & $0.2360(5)$ & $0.050(5)$ & $0.139(8)$ & $0.082(6)$ & $-0.016(5)$ & $0.007(4)$ & $-0.058(5)$ \\
\hline $\mathrm{N}(2)$ & $8 e$ & $0.6013(3)$ & $0.138(1)$ & $0.3706(4)$ & $0.045(4)$ & $0.066(5)$ & $0.048(5)$ & $0.011(4)$ & $0.008(4)$ & $0.003(4)$ \\
\hline$N(3)$ & $8 e$ & $0.6791(3)$ & $0.449(1)$ & $0.4645(4)$ & $0.047(4)$ & $0.080(5)$ & $0.052(4)$ & $0.018(4)$ & $0.000(3)$ & $-0.003(4)$ \\
\hline $\mathrm{O}(1)$ & $8 e$ & $0.6279(4)$ & $0.6000(9)$ & $0.1777(4)$ & $0.088(5)$ & $0.119(7)$ & $0.071(5)$ & $0.005(4)$ & $0.004(4)$ & $-0.036(4)$ \\
\hline $\mathrm{O}(2)$ & $8 e$ & $0.6858(4)$ & $0.396(1)$ & $0.0863(4)$ & $0.087(5)$ & $0.109(6)$ & $0.083(5)$ & $0.029(4)$ & $0.004(4)$ & $-0.007(4)$ \\
\hline $\mathrm{O}(3)$ & $8 e$ & $0.6579(8)$ & $0.679(1)$ & $0.0424(7)$ & $0.48(2)$ & $0.084(7)$ & $0.19(1)$ & $0.05(1)$ & $0.15(1)$ & $0.072(8)$ \\
\hline $\mathrm{O}(4)$ & $8 e$ & $0.5676(5)$ & $0.477(2)$ & $0.0692(6)$ & $0.080(6)$ & $0.43(2)$ & $0.109(7)$ & $0.057(8)$ & $-0.037(5)$ & $-0.13(1)$ \\
\hline$C(1)$ & $8 e$ & $0.6211(5)$ & $0.059(1)$ & $0.2240(5)$ & $0.079(7)$ & $0.093(8)$ & $0.046(6)$ & $-0.016(6)$ & $0.000(5)$ & $-0.008(5)$ \\
\hline $\mathrm{C}(2)$ & $8 e$ & $0.5737(5)$ & $0.033(1)$ & $0.2984(6)$ & $0.045(5)$ & $0.119(8)$ & $0.065(7)$ & $-0.005(5)$ & $-0.003(5)$ & $-0.014(6)$ \\
\hline $\mathrm{C}(3)$ & $8 e$ & $0.5814(5)$ & $0.335(1)$ & $0.3662(5)$ & $0.053(5)$ & $0.067(7)$ & $0.068(6)$ & $0.010(5)$ & $-0.001(5)$ & $0.002(5)$ \\
\hline $\mathrm{C}(4)$ & $8 e$ & $0.5993(4)$ & $0.445(1)$ & $0.4454(6)$ & $0.051(6)$ & $0.088(7)$ & $0.065(6)$ & $0.016(5)$ & $0.010(5)$ & $-0.005(6)$ \\
\hline
\end{tabular}

Acknowledgments. The authors thank the Education Office of Hubei Province, P. R.China, for a research grant (No. 2002B29002) and the Natural Science Foundation of Hubei Province, P.R.China (grant No. 2003ABB010).

\section{References}

1. Ren, C.-X.; Zhu, H.-L.; Yang, G.; Chen, X.-M.: Syntheses and crystal structures of five two-dimensional networks constructed from staircase-like silver(I) thiocyanate chains and bridging polyamines. J. Chem. Soc., Dalton Trans. (2001) 85-90.

2. Sheldrick, G. M.: Siemens SHELXTL (Version 5.0): Siemens Industrial Automation, Inc., analytical Instrumentation, USA 1995.

3. Sheldrick, G. M.: SHELXTL-plus. Release 4.1. Siemens Analytical X-ray Instruments Inc., Madison Wisconsin, USA 1991. 